\title{
3D Multi-source Least-squares Reverse Time Migration
}

Wei Dai, C. Boonyasiriwat and Gerard T. Schuster, King Abdullah University of Science and Technology

\section{SUMMARY}

We present the theory and numerical results for least-squares reverse time migration (LSRTM) of phase-encoded supergathers, where each supergather is the superposition of phasedencoded shots. Three type of encoding functions are used in this study: random time shift, random source polarity and random source location selected from a pre-designed table. $\mathrm{Nu}-$ merical tests for the 3D SEG/EAGE Overthrust model show that multi-source LSRTM can suppress migration artifacts in the migration image and remove most of the crosstalk noise from multi-source data. Empirical results suggest that multisource LSRTM can provide a noticeable increase in computational efficiency compared to standard RTM, when the CSGs in a supergather are modeled and migrated together with a finite-difference simulator. If the phase-encoding functions are dynamically changed after each iteration of LSRTM, the best images are obtained. The potential drawback is that the final results are very sensitive to the accuracy of the starting model.

\section{INTRODUCTION}

Data acquisition with multiple sources requires the excitation of $N$ phase-encoded shots at the same time and recording of the resulting wavefields. The recorded data will be denoted as an $\mathrm{N}$-source supergather. This topic has recently become popular because it reduces the economic cost of seismic acquisition, especially for the cases of VSP, OBC and OBS data acquisition (Akerberg et al., 2008; Beasley, 2008; Berkhout, 2008; Fromyr et al., 2008; Hampson et al., 2008; Huo et al., 2009; Kim et al., 2009; Moore et al., 2008; Spitz et al., 2008). For wide azimuth acquisition, multiple vessels are used, so it is convenient to perform multiple source shooting.

Many methods have been proposed to separate a supergather into individual shot gathers (Akerberg et al., 2008; Beasley, 2008; Berkhout, 2008; Fromyr et al., 2008; Moore et al., 2008; Spitz et al., 2008). The simplest approach is to perform separation by the standard migration and stacking procedures. Lynn et al. (1987) showed that it is effective to remove coherent noise in multi-source data by weighted stacking. Beasley (2008) presented his previous work and showed that the cross-talk noise was attenuated by standard migration and stacking for 2 -source supergathers. Fromyr et al. (2008) achieved image quality with 2 -source shooting similar to that from conventional acquisition in their wide azimuth experiment. Berkhout (2008) extended the simultaneous sources acquisition to blended acquisition and presented his theoretical work; he also showed that standard stacking and migration can suppress the crosstalk noise quite well. With careful survey design, a suitable marine environment and a small number of multiple sources, simple stacking alone is sufficient for quality imaging.

Another important application of multi-source acquisition is in waveform inversion. Zhan et al. (2009) applied multi-source multi-scale waveform inversion to synthetic data and used a deblurring filter to reduce the crosstalk error and speed up convergence. Their numerical results showed a 12-fold speed up in computation efficiency. Krebs et al. (2009) presented their waveform inversion result with simultaneous sources encoded by random encoding functions. Their computational efficiency was increased by a factor of 50 compared to conventional inversion. Both of the above results were for $2 \mathrm{D}$ simulations, but in the 3D case, with one extra order of freedom more sources can be used to construct a supergather to achieve an even higher efficiency.

In this paper, the theory of iterative least-squares migration for processing supergathers is presented. With multi-source LSRTM, the size of the data space is reduced by $N$ times ( $N$ being the number of multiple sources) and so ameliorates the burden of onerous I/O demands. For 3D prestack migration, significantly reducing the large I/O costs will lead to a significant reduction in clocktime.

\section{THEORY}

The forward modeling of scatterred wavefield under the Born approximation is described as

$$
\mathbf{d}(\mathbf{g} \mid \mathbf{x})=2 \omega^{2} \int_{\mathbf{x}} G(\mathbf{g} \mid \mathbf{x}) G(\mathbf{x} \mid \mathbf{s}) \mathbf{m}(\mathbf{x}) d \mathbf{x},
$$

where $G(\mathbf{g} \mid \mathbf{x})$ is the Green's function for a source at $\mathbf{x}$ and geophone at $\mathbf{g}$. Equation 1 can be rewritten in matrix notation as

$$
\mathbf{d}_{i}=\mathbf{L}_{i} \mathbf{m},
$$

where $\mathbf{L}$ is the forward modeling operator. The goal of seismic imaging is to migrate the data $\mathbf{d}$ to get the estimate of the reflectivity image;

$$
\mathbf{m}(\mathbf{x})=2 \omega^{2} \int_{\mathbf{s}, \mathbf{g}} G(\mathbf{g} \mid \mathbf{x})^{*} G(\mathbf{x} \mid \mathbf{s})^{*} \mathbf{d}(\mathbf{g} \mid \mathbf{s}) d \mathbf{s} d \mathbf{g},
$$

or in matrix notation

$$
\mathbf{m}=\mathbf{L}^{T} \mathbf{d}
$$

Recently, the method of multi-source imaging has emerged, where many phase-encoded shot are excited and a supergather is recorded as

$$
\mathbf{d}=\sum_{i=1}^{N} \mathbf{S}_{i} \mathbf{d}_{i}
$$

where $N$ represents the number of multiple shots, and $\mathbf{S}_{i}$ represents the phase-encoding functions. All $\mathbf{S}_{i}$ are unitary and $\mathbf{d}_{i}$ represents individual shot gathers;

$$
\mathbf{d}_{i}=\mathbf{L}_{i} \mathbf{m} \text {. }
$$

The multi-source forward modeling operator $\mathbf{L}$ and multi-source migration operator $\mathbf{L}^{T}$ are defined as

$$
\mathbf{L}=\sum_{i=1}^{N} \mathbf{S}_{i} \mathbf{L}_{i}, \mathbf{L}^{T}=\sum_{i=1}^{N} \mathbf{L}_{i}^{T} \mathbf{S}_{i}^{T},
$$




\section{D Multi-source LSRTM}

so that the migration image becomes

$$
\begin{aligned}
\mathbf{m}_{\text {mig }} & =\mathbf{L}^{T} \mathbf{d}=\mathbf{L}^{T} \sum_{i=1}^{N} \mathbf{S}_{i} \mathbf{L}_{i} \mathbf{m}, \\
& =\sum_{j=1}^{N} \mathbf{L}_{j}^{T} \mathbf{S}_{j}^{T} \sum_{i=1}^{N} \mathbf{S}_{i} \mathbf{L}_{i} \mathbf{m}, \\
& =\sum_{i=1}^{N} \sum_{j=1}^{N} \mathbf{L}_{j}^{T} \mathbf{S}_{j}^{T} \mathbf{S}_{i} \mathbf{L}_{i} \mathbf{m}, \\
& =\overbrace{\sum_{i=1}^{N} \mathbf{L}_{i}^{T} \mathbf{L}_{i} \mathbf{m}}^{\text {migration image }}+\overbrace{\sum_{j \neq i} \sum_{i=1}^{N} \mathbf{L}_{j}^{T} \mathbf{S}_{j}^{T} \mathbf{S}_{i} \mathbf{L}_{i} \mathbf{m}}^{\text {crosstalk }},
\end{aligned}
$$

which consists of two terms. The first term is the standard migration image and the second term is the crosstalk noise introduced by migrating blended multi-source data.

In order to suppress crosstalk noise to an acceptable level when the number of multiple sources $N$ is large, the least-squares migration is used (Dai and Schuster, 2009; Schuster, 2009). The starting point is to define the objective function

$$
f(\mathbf{m})=\frac{1}{2}\|\mathbf{d}-\mathbf{L m}\|^{2},
$$

so that an optimal $\mathbf{m}$ is sought to minimize the objective function. The optimal solution is found by a gradient type optimization method, e.g.,

$$
\mathbf{m}^{(k+1)}=\mathbf{m}^{(k)}-\alpha \mathbf{F} \mathbf{L}^{T}\left(\mathbf{L} \mathbf{m}^{(k)}-\mathbf{d}\right),
$$

where $\mathbf{F}$ is a preconditioner (Nemeth et al., 1999), and $\alpha$ is the step length calculated from back-tracking and a numerical line search. The conjugate gradient method is used, which generally converges faster than the steepest decent method. After each iteration, the phase-encoding functions can be kept the same (static encoding) or dynamically changed (dynamic encoding). Numerical results will be shown that suggests that dynamic encoding is the best strategy for suppressing crosstalk. Three types of encoding functions will be used: random time shifts, random source polarities, and random source locations selected from a table which contains all the predetermined source locations.

In this study, the reverse time migration operator is used as $\mathbf{L}^{T}$, but the Born modeling operator is too expensive in the sense that all the Green's functions need to be saved and convolution is required at each image point. The finite-difference simulation is computed twice with slightly different models and the difference between the two recorded wavefields is calculated as the scattered field,

$$
\mathbf{L m}=\mathbf{F}\left(\mathbf{s}_{0}+\mathbf{m}\right)-\mathbf{F} \mathbf{s}_{0},
$$

where $\mathbf{s}_{0}$ represents the slowness of a smooth background model and $\mathbf{m}$ is the reflectivity model, which represents a slowness perturbation in above equation.

\section{RESULTS}

The multi-source LSRTM algorithm is tested on the synthetic data generated by FD (finite-difference) solutions to the 3D acoustic wave equation for the 3D SEG/EAGE Overthrust model. The model size is $800 \times 800 \times 186$ and Figure 1 shows the verti$\mathrm{cal}$ and horizontal slices of the true model. Figure 2 shows the starting velocity model, which is also the background velocity model kept constant during iterations.

There are 1089 shots and 10201 receivers evenly deployed on the surface. We separate the 1089 shots into 11 supergathers with 99 shot gathers per supergather. A 2-4 finite-difference simulator is used to solve the acoustic wave equation with a 20 $\mathrm{m}$ grid interval and a $5 \mathrm{~Hz}$ source wavelet. All the numerical tests are performed with 4096 CPUs on an IBM Blue Gene supercomputer. Figure 3 shows the vertical and horizontal slices of the multi-source RTM image. Compared to the conventional RTM, the multi-source RTM is 99 times more computationally efficient. For the 3D model used in this study, the conventional RTM image would take about 20 days to compute compared to the only $5 \mathrm{CPU}$ hours for the multi-source result. However, the single iteration LSRTM result has much less quality than the conventional RTM image.

For the next step, multi-source least-squares migration is carried out with both static encoding and dynamic encoding for comparison. Figure 4 shows the multi-source LSRTM images after 10 iterations with static encoding and Figure 5 shows the result with dynamic encoding after 10 iterations. Compared to Figure 3, the multi-source LSRTM image in Figure 4 shows fewer artifacts associated with the source locations and a more balanced amplitude in the vertical slice; in the horizontal slice, the crosstalk noise is attenuated. In Figure 5, the best result is presented. When dynamic encoding is used, the multisource LSRTM images show the least noise. Figure 6 shows the convergence curves for both the static and dynamic encoding methods, where both methods show similar convergence rate.

\section{CONCLUSION}

Multi-source least-squares reverse time migration with phase encoding is applied to 3D supergathers. Numerical tests show that iterative LSM helps remove migration artifacts and suppress crosstalk when shots gathers are blended and migrated at the same time. When comparing the results from static and dynamic encoding, we found that the dynamic encoding method produces a result with the best $\mathrm{S} / \mathrm{N}$ ratio.

\section{ACKNOWLEDGMENTS}

We thank the staff at KAUST HPC for technical support. 


\section{D Multi-source LSRTM}
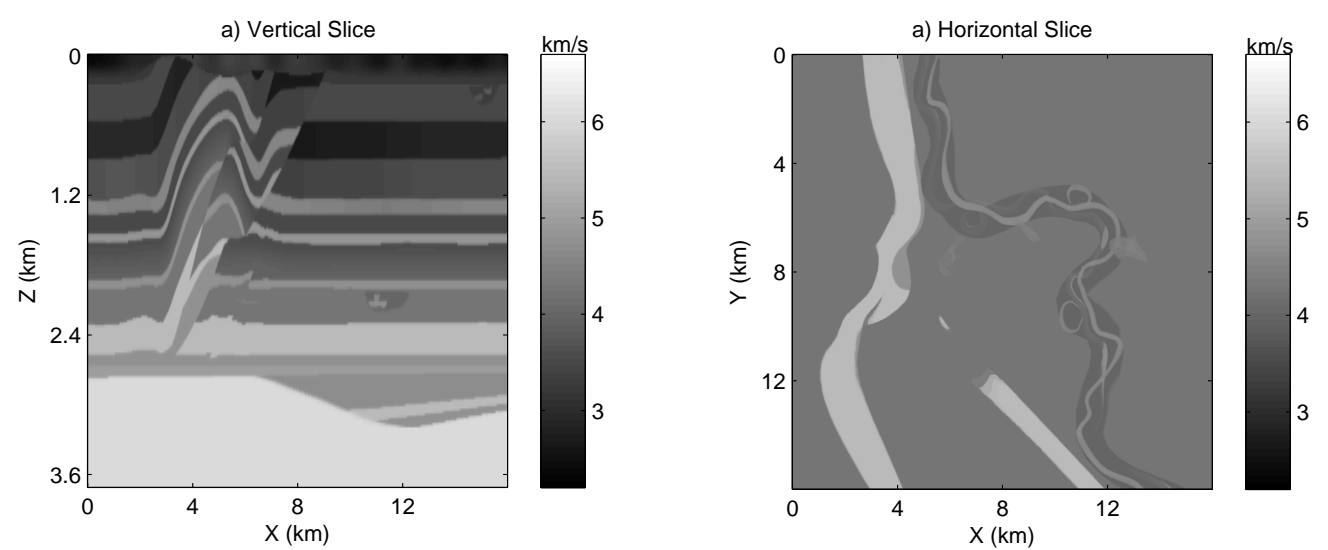

Figure 1: a). Vertical slice $(y=8000 \mathrm{~m})$ and $\mathrm{b})$. horizontal slice $(\mathrm{z}=2100 \mathrm{~m})$ of the 3D SEG/EAGE Overthrust model.
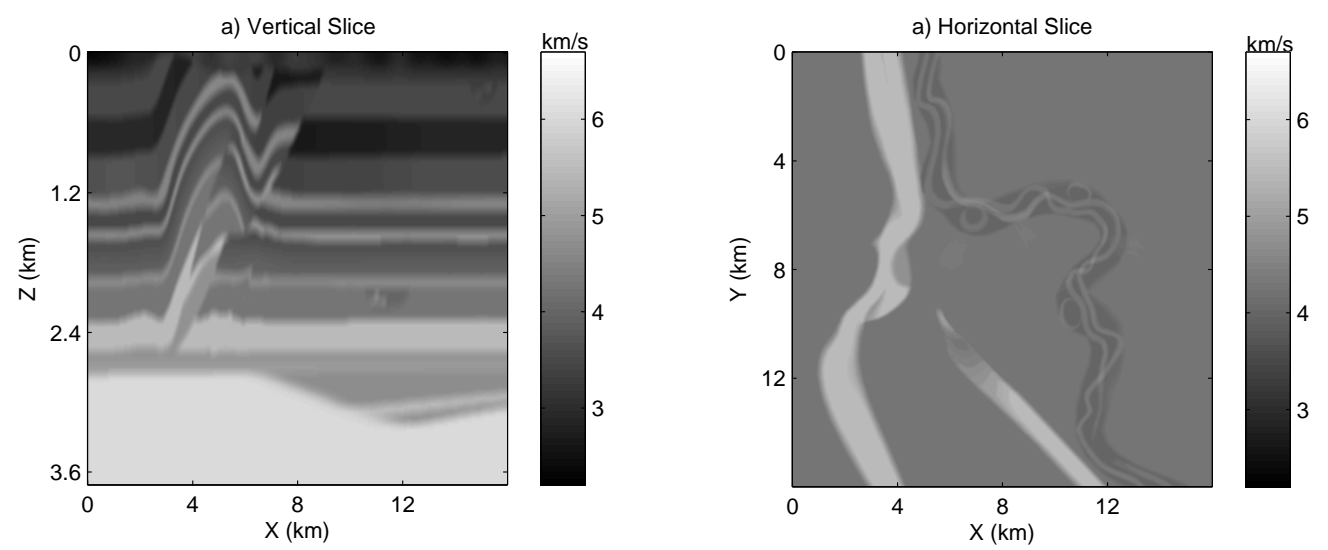

Figure 2: a). Vertical slice $(\mathrm{y}=8000 \mathrm{~m})$ and $\mathrm{b})$. horizontal $(\mathrm{z}=2100 \mathrm{~m})$ of the background velocity model.
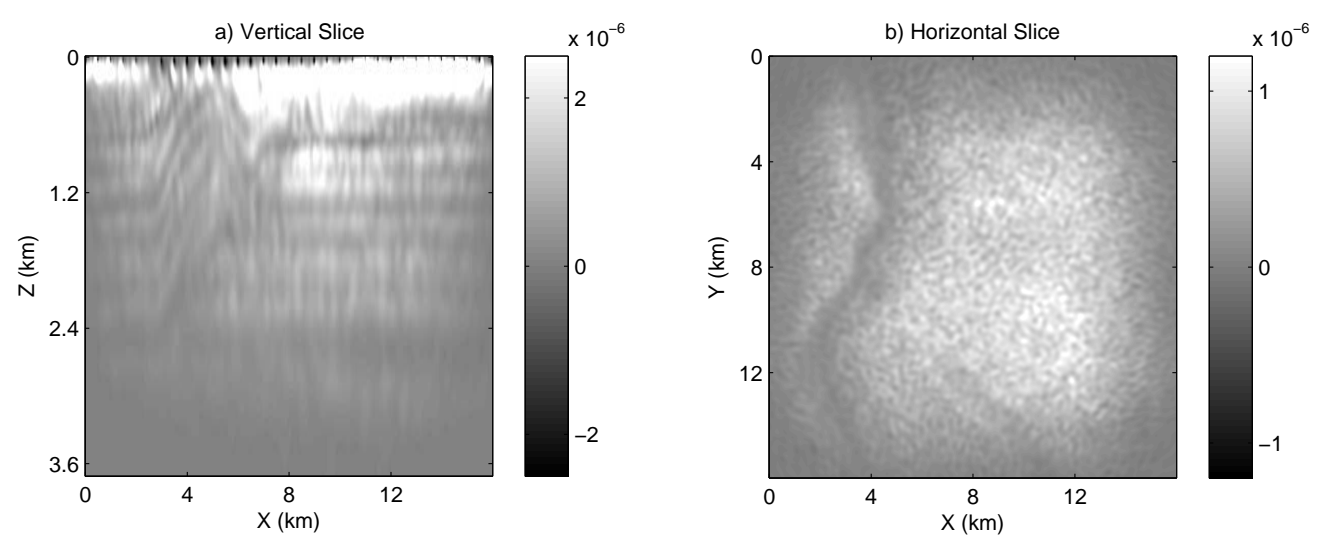

Figure 3: a). Vertical slice $(y=8000 m)$ and $b)$. horizontal slice $(z=2100 m)$ of the multi-source reverse time migration image. 


\section{D Multi-source LSRTM}
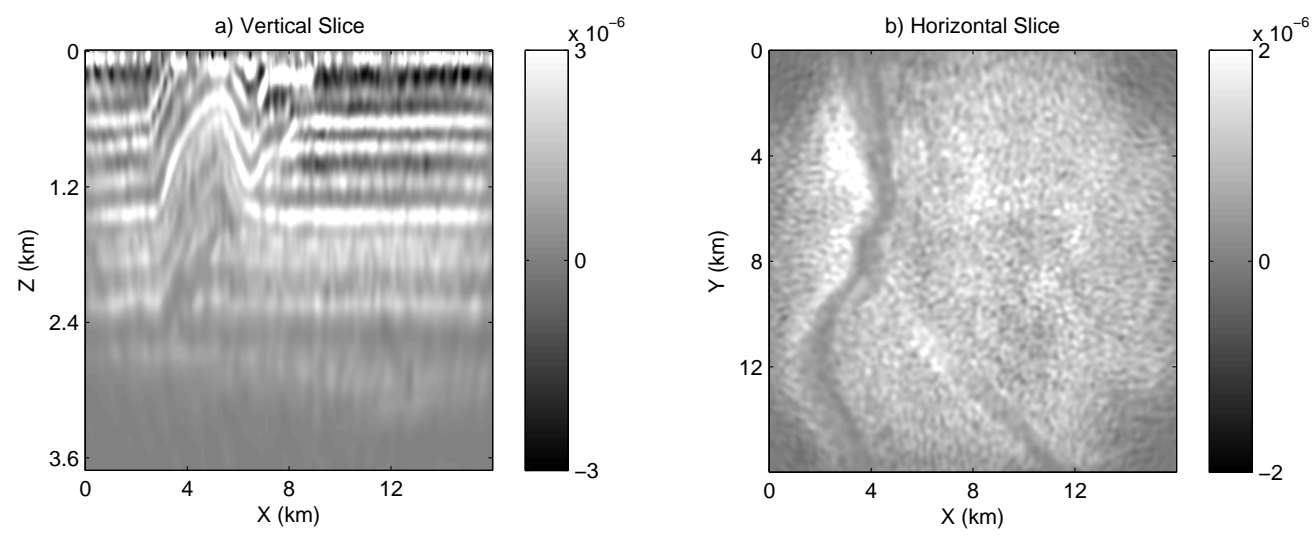

Figure 4: a). Vertical slice $(y=8000 \mathrm{~m})$ and $\mathrm{b})$. horizontal slice $(\mathrm{z}=2100 \mathrm{~m})$ of the multi-source least-squares reverse time migration image with static encoding after 10 iterations.
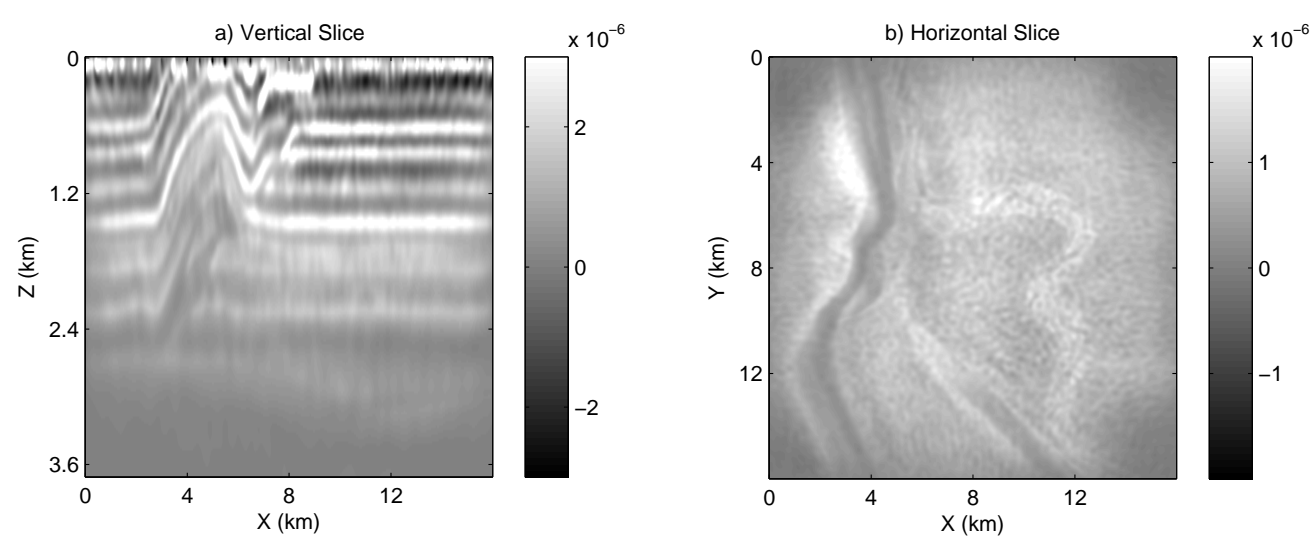

Figure 5: a). Vertical slice $(y=8000 \mathrm{~m})$ and $\mathrm{b})$. horizontal slice $(\mathrm{z}=2100 \mathrm{~m})$ of the multi-source least-squares reverse time migration image with dynamic encoding after 10 iterations.

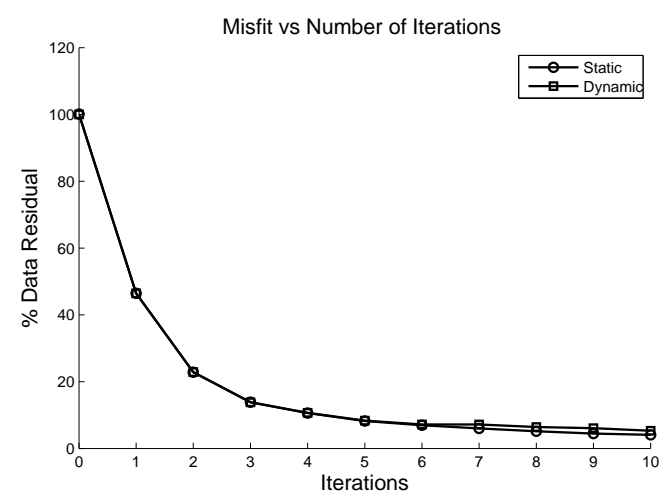

Figure 6: Data residual vs iterations for multi-source least-squares reverse time migration with static encoding (circles) and dynamic encoding (squares). 


\section{EDITED REFERENCES}

Note: This reference list is a copy-edited version of the reference list submitted by the author. Reference lists for the 2010 SEG Technical Program Expanded Abstracts have been copy edited so that references provided with the online metadata for each paper will achieve a high degree of linking to cited sources that appear on the Web.

\section{REFERENCES}

Akerberg, P., G. Hampson, J. Rickett, H. Martin, and J. Cole, 2008, Simultaneous source separation by sparse radon transform: SEG Technical Program Expanded Abstracts, 27, no. 1, 2801-2805, doi:10.1190/1.3063927.

Beasley, C. J., 2008, A new look at marine simultaneous sources: The Leading Edge, 27, no. 7, 914-917, doi:10.1190/1.2954033.

Berkhout, A. J. G., 2008, Changing the mindset in seismic data acquisition: The Leading Edge, 27, no. 7, 924-938, doi:10.1190/1.2954035.

Dai, W., and G. T. Schuster, 2009, Least-squares migration of simultaneous sources data with a deblurring filter: SEG Technical Program Expanded Abstracts, 28, 2990-2994.

Fromyr, E., G. Cambois, R. Loyd, and J. Kinkead, 2008, Flam - a simultaneous source wide azimuth test: SEG Technical Program Expanded Abstracts, 27, no. 1, 2821-2825, doi:10.1190/1.3063931.

Hampson, G., J. Stefani, and F. Herkenhoff, 2008, Acquisition using simultaneous sources: The Leading Edge, 27, no. 7, 918-923, doi:10.1190/1.2954034.

Huo, S., Y. Luo, and P. Kelamis, 2009, Simultaneous sources separation via multidirectional vectormedian filter: SEG Technical Program Expanded Abstracts, 28, 31-35.

Kim, Y., I. Gruzinov, M. Guo, and S. Sen, 2009, Source separation of simultaneous source obc data: SEG Technical Program Expanded Abstracts, 28, 51-55.

Krebs, J. R., J. E. Anderson, D. Hinkley, R. Neelamani, S. Lee, A. Baumstein, and M.-D. Lacasse, 2009, Fast full-wavefield seismic inversion using encoded sources: Geophysics, 74, no. 6, WCC177WCC188, doi:10.1190/1.3230502.

Lynn, W., M. Doyle, K. Larner, and R. Marschall, 1987, Experimental investigation of interference from other seismic crews: Geophysics, 52, 1501-1524, doi:10.1190/1.1442268.

Moore, I., B. Dragoset, T. Ommundsen, D. Wilson, C. Ward, and D. Eke, 2008, Simultaneous source separation using dithered sources: SEG Technical Program Expanded Abstracts, 27, no. 1, 28062810, doi:10.1190/1.3063928.

Nemeth, T., C. Wu, and G. T. Schuster, 1999, Least-squares migration of incomplete reflection data: Geophysics, 64, 208-221, doi:10.1190/1.1444517.

Schuster, G. T., 2009, Multi-source least squares migration, waveform inversion, and mva analysis: SEG Invited Workshop Talk.

Spitz, S., G. Hampson, and A. Pica, 2008, Simultaneous source separation: A prediction-subtraction approach: SEG Technical Program Expanded Abstracts, 27, no. 1, 2811-2815, doi:10.1190/1.3063929.

Zhan, G., C. Boonyasiriwat, W. Dai, and G. T. Schuster, 2009, Multi-source waveform inversion with deblurring: Journal of Seismic Exploration. 